\title{
A Cosmological Solution to the Impossibly Early Galaxy Problem
}

\author{
Manoj K. Yennapureddy ${ }^{1 \mathrm{a}}$, Fulvio Melia ${ }^{2 \mathrm{~b}}$ \\ ${ }^{a}$ Department of Physics, The University of Arizona, AZ 85721, USA \\ ${ }^{b}$ Department of Physics, The Applied Math Program, and Department of Astronomy, \\ The University of Arizona, AZ 85721, USA
}

\begin{abstract}
To understand the formation and evolution of galaxies at redshifts $0 \lesssim z \lesssim$ 10, one must invariably introduce specific models (e.g., for the star formation) in order to fully interpret the data. Unfortunately, this tends to render the analysis compliant to the theory and its assumptions, so consensus is still somewhat elusive. Nonetheless, the surprisingly early appearance of massive galaxies challenges the standard model, and the halo mass function estimated from galaxy surveys at $z \gtrsim 4$ appears to be inconsistent with the predictions of $\Lambda$ CDM, giving rise to what has been termed "The Impossibly Early Galaxy Problem" by some workers in the field. A simple resolution to this question may not be forthcoming. The situation with the halos themselves, however, is more straightforward and, in this paper, we use linear perturbation theory to derive the halo mass function over the redshift range $0 \lesssim z \lesssim 10$ for the $R_{\mathrm{h}}=c t$ universe. We use this predicted halo distribution to demonstrate that both its dependence on mass and its very weak dependence on redshift are compatible with the data. The difficulties with $\Lambda$ CDM may eventually be overcome with refinements to the underlying theory of star formation and galaxy evolution within the halos. For now, however, we demonstrate that the unexpected early formation of structure may also simply be due to an incorrect choice of the cosmology, rather than to yet unknown astrophysical issues associated with the condensation of mass fluctuations and subsequent galaxy formation.
\end{abstract}

Keywords: cosmological parameters, cosmological observations,

\footnotetext{
${ }^{1}$ E-mail: manojy@email.arizona.edu

${ }^{2}$ John Woodruff Simpson Fellow. E-mail: fmelia@email.arizona.edu
} 
cosmological theory, dark energy, galaxies, large-scale structure

\section{Introduction}

The structures we see today are believed to have grown gravitationally from tiny fluctuations in the primordial density field. Current theory holds that perturbations started to collapse once their density exceeded a certain critical value, forming bound objects that then assembled together with the surrounding gas and dust to form stars, galaxies and clusters. With dark matter particles decoupling first from the radiation, the early stages of structure formation proceeded principally through the condensation of dark matter halos. Baryonic particles subsequently accreted into the potential valleys created in this fashion once they themselves decoupled from the relativistic background.

The physics responsible for the formation of galaxies in this scenario is still not completely understood, but there is general consensus concerning the rate at which halos formed, specifically their number density distribution as a function of mass and redshift $[1,2,3]$. This halo mass function (as it is more commonly known) was first derived analytically by Press \& Schechter [4] using several simplifying assumptions, including a spherically symmetric collapse model and a Gaussian initial density field. But though this analysis predicts a reasonable distribution, it nonetheless also underpredicts the number of high-mass halos and overpredicts the low-mass ones compared to detailed numerical simulations. More recently, Sheth \& Tormen [1] have shown that this discrepancy may be mitigated by adopting an ellipsoidal collapse model rather than spherical. Even so, these analytical and semi-analytical approaches have for the most part been tested only against numerical simulations. Unfortunately, while Press-Schechter underpredicts the number of high-mass halos, Sheth-Tormen apparently overpredicts them, though a correction factor based on the linear growth rate may have been found. We shall describe this effect following Equation (21) below. It is more difficult to test these semi-analytic approaches using actual observations because halos cannot be seen directly. The predicted halo distribution must be compared to the data indirectly, through the observation of the galaxy mass function, with an added assumption concerning the evolutionary relationship between them.

The observed halo formation was recently assessed [5] using several previous analyses to compare different techniques for relating the halo and galaxy 
distributions. For this purpose, these authors employed high redshift surveys in the redshift range $z \sim 4-8$, principally The Cosmic Assembly Near-infrared Deep Extragalactic Survey (CANDELS [6, 7]) and the Spitzer Large Area Survey with Hyper-Suprime-Cam (SPLASH [8]), to probe the galaxy luminosity and mass functions, from which the halo distribution may be derived. CANDELS is well suited to find the lower-mass galaxies because it represents a survey over a small area, whereas SPLASH has broad sky coverage and can therefore probe the more massive galaxies.

Obtaining the halo distribution and masses from the galaxy distribution presents quite a challenge. The best way to obtain halo masses from the spatial distribution of galaxies is via galaxy clustering methods $[9,10]$, which don't assume any physical properties of the galaxies themselves, though they must assume a model for the dark matter concentration. Other techniques use the relationship between the luminosity and stellar masses, obtained from template fitting [11]. For example the "abundance matching technique" [12] relates one of the key features in the luminosity or mass function, such as the knee, to a feature in the halo mass function, and then matches the galaxy density and dark-matter halo density to derive halo masses over the whole mass range. Alternatively, one may also assume that the relations derived at low redshift using luminosity to dark-matter mass ratios still apply at high redshifts.

But though each of these techniques yields somewhat different outcomes in a quantitative sense, they all agree qualitatively [5]. These earlier findings show quite emphatically that the halo distribution estimated from galaxies at $z \gtrsim 4$ in the CANDELS and SPLASH surveys is inconsistent with the evolution of the halo mass function and the galaxy luminosity and mass functions predicted by standard $\Lambda \mathrm{CDM}[9,10,13,12]$ - a situation termed "The Impossibly Early Galaxy Problem" [5]. Various possible remedies were considered by these authors to reconcile the observed and predicted halo mass distributions, including possible errors introduced in calibrating the data using relations derived at lower redshifts, which may not be applicable for $z \gtrsim 4$. None of the remedies worked, however. If anything, this extended study showed that the high-redshift galaxies appear normal, suggesting that the relations derived at lower redshifts are probably also applicable at these higher redshifts.

The tension between the predicitons of $\Lambda \mathrm{CDM}$ and the 'measured' halo mass function may be resolved with a better understanding of the underlying physics, e.g., regarding start formation and galaxy evolution. On the 
other hand, the current uncertain situation may simply be an indication that there are insurmountable problems with the use of $\Lambda \mathrm{CDM}$ as the background cosmology. In this paper, we will proceed under this assumption-i.e., that the problems elucidated by Steinhardt et al. [5] are real, and seek to find a solution to the surprisingly early formation of massive halos. To balance the discussion, however, we acknowledge the fact that this point of view is not universally accepted - a situation largely due to uncertainties in the simulations used to fully interpret the data and halo mass function.

Understanding the evolution of galaxies and their observational signatures, such as their UV luminosity or their redshift-dependent clustering, necessarily relies on modeling dark-matter evolution [14], and cosmological hydrodynamical simulations $[15,16]$, complemented by analytical and semianalytical calculations $[17,18]$. Complications arise in part because the observed UV luminosity function depends strongly on redshift (at least from $z \sim 4$ to 10), and various combinations of inputs and assumptions produce degenerate results $[19,20]$. It is fair to say that the degree of tension between the observations and predictions of the standard model depends on one's point of view.

But as noted, there are good reasons to suspect that real problems with the formation of structure do exist in the standard model. Some of these have to do with the unusually early appearance of supermassive black holes at $z \sim 6-7[21,22]$ and galaxies at $z \sim 10-12$ (see refs. cited in [23]). In addition, a rather compelling case may be made that a problem exists [5] based on the following points: (1) the halo mass function at $0 \lesssim z \lesssim 8$ is inferred using 3 or 4 different techniques, not just one, and all of the results agree at least qualitatively; (2) the fact that these techniques all require a blending of observational and simulational (i.e.,model-dependent ) factors to arrive at a mutually consistent picture meansre that it is difficult to understand exactly what the results mean, because such an approach is very compliant to the assumptions one makes. For example, abundance matching forces agreement between observation and theory even in the absence of a strong physical motivation for the underlying model. The uncertainties (e.g., in how to match star-forming galaxy UV luminosities with halo formation in both mass and time) leave unresolved questions concerning how galactic evolution impacts our understanding of halo evolution. Nonetheless, forcing consistency between the observations and predictions of the standard model comes at a considerable cost.

One may understand this situation as follows. Much of the analysis in 
this paper is based on the 'standard' ratio of halo to stellar-mass, which arises from two considerations: First is the expectation that $10 \%$ of the baryonic matter condensed into stars [24]. Second, is the ratio of dark matter to baryonic matter, which is observed to be about 6:1 [25]. As we shall detail below, Steinhardt et al. [5] attempted to reconcile the disparity between theory and observation by introducing several modifications to the underlying physical processes. In order to fit the derived halo mass function in $\Lambda$ CDM, however, they found that only a change by 0.8 dex in the ratio of dark matter to baryonic matter would suffice. But such a drastic change could come about only with a complete absence of dark matter at redshift 8 , or if essentially $100 \%$ of the baryons condensed into stars at higher redshifts. Both of these scenarios constitute implausible physics, such as the need to convert all of the baryons into stars instantly upon halo virialization [5]. Other attempted remedies have equally unlikely requirements. So perhaps a better way to characterize the problem with the halo mass function is to say that it can only be made consistent with expectations of the standard model with the adoption of unlikely, new physics.

Given the unsettled debate concerning the formation and evolution of galaxies, we stress that our focus in this paper is not to model the galaxies themselves. We merely use some key observations of galactic profiles to infer the mass and time evolution of halos which, in principle, constitutes a much simpler, cleaner objective. For a complete assessment of problems with the formation of structure, it will eventually be necessary to study both the formation of halos and the galaxies within them, but this is a much more challenging analysis than we are attempting here. Such elaborate simulations for the formation and clustering of galaxies are outside the scope of the present paper. The outcome of this subsequent work will be reported elsewhere.

In the present context, the difficulty that $\Lambda$ CDM has in accounting for the observed halo mass function has much in common with the growing tension between the measured cosmological growth rate, $b \sigma_{8}(z)$, and its value predicted by the standard model, particularly in the redshift range $0<z<1$, where a significant curvature expected in the functional form of $b \sigma_{8}(z)$ is absent in the data [26]. Admittedly, the errors in the measured values of $b \sigma_{8}(z)$ are still too large to rule out any model, but this is precisely why a comparison of the measured halo mass function with theory is very probative. If it turns out that both the halo distribution at high redshift and $b \sigma_{8}(z)$ at lower redshift are in tension with the growth of structure expected in standard 
cosmology, a compelling argument can be made that an alternative expansion scenario must be seriously considered. In this paper, we therefore compare the measured halo mass function, not only with the prediction of $\Lambda$ CDM, but also with that expected in the alternative Friedmann-Robertson-Walker (FRW) cosmology known as the $R_{\mathrm{h}}=c t$ universe [27, 28, 29, 30, 31]. It was a direct one-on-one comparison between these two models that highlighted the greater consistency of the growth rate data with $R_{\mathrm{h}}=c t$ than with $\Lambda$ CDM [26]. As we shall see shortly, the measured halo mass function also provides strong support for $R_{\mathrm{h}}=c t$ and, if the tension between the predictions of $\Lambda \mathrm{CDM}$ and the data fail to be resolved, may also eventually argue that $R_{\mathrm{h}}=c t$ is favoured over the standard model.

\section{The Halo Mass Function in $\Lambda \mathrm{CDM}$}

Let us first see how the halo mass function predicted by the standard model fares in comparison with the data. Throughout this paper, we use the number density and halo mass calibrated in ref. [5] (and references cited therein) based on the CANDELS and SPLASH surveys. These data, along with seven theoretical curves calculated within the redshift range $z=4-10$, are shown in figure 1 . The theoretical curves in this figure are based on the halo mass function estimates of Sheth \& Tormen [1], using the HMFCalc code developed in ref. [32]. The standard model parameters are assumed to have their Planck values, $\left(h, \Omega_{\mathrm{m}}, \Omega_{\Lambda}\right)=(0.704,0.272,0.728)[25]$. Some of these data points were obtained using the clustering technique and the photometric spectral energy distribution (SED) based on template fitting. A ratio $M_{H} / M_{*} \sim 70$ [24] was used to convert the stellar mass function $M_{*}$ to a halo mass $M_{H}$. For the data points obtained using the UV luminosity function, a conversion $M_{H} / M_{\odot} \approx 120 L_{U V} / L_{\odot}$ was used to convert UV luminosity to stellar mass and then to halo mass.

The assumption of a constant ratio $M_{H} / M_{*} \sim 70$ (and correspondingly for the UV luminosity) can have a strong influence on the analysis in this paper, so it is appropriate to question its reliability. The situation today is somewhat unfortunate in this regard, given that adequate data are lacking to determine empirically what these ratios are-indeed, whether they are even constant with redshift. And simulations carried out by several groups do not appear to produced fully consistent outcomes. We ourselves do not have a position in this discussion, and eagerly await a resolution in order to put our calculations on firmer ground. For the time being, our goal is to find the 
most reasonable compromise, while maintaining a manageable approach to avoid tainting our results with excessive (possibly incorrect) detail.

There is some evidence that this ratio is more or less constant based on fits to the inferred halo to stellar mass ratio at lower redshifts, as explained in ref. [24], but its adoption is not universally accepted. There has been some debate $[33,34,10,35,36]$ regarding whether or not these numbers remain unchanged at higher redshifts. Not surprisingly, there is therefore disagreement between various researchers regarding whether or not the halo to stellar mass relation should be parameterized in terms of both mass and redshift, rather than just the mass or redshift on its own. For example, refs. [41, 17] used both mass and redshift, a conclusion supported by the simulations of Finkelstein et al. [12], who reported that the halo to stellar mass in their calculations evolves with both mass and redshift. For example, their results showed that, at constant UV luminosity, the stellar-to-halo mass ratio increases with $z$. This trend was also inferred in ref. [24], where the halo mass function was parametrized in terms of $z$. But on the other side of this debate, refs. [37, 38] reported that the median stellar mass to halo mass relation does not evolve strongly in the redshift range $5 \lesssim z \lesssim 12$. Some additional uncertainty was generated by the work in ref. [18], where quite a different approach was used to match the observations with theory. These workers abandoned the need for continuous evolution altogether, and instead allowed mass to be both added and subtracted in order to allow the halo mass function to match the available data at each redshift.

There may be an explanation for why there exist two camps in this discussion. It is certainly true that the ratio of halo mass to luminosity varies in the simulations of refs. [12, 38, 37, 39, 40], who reported an evolution in both UV luminosity and redshift. Others have pointed out several caveats, however, mostly having to do with the fact that these conclusions are based primarily on theoretical modeling. But one cannot always be certain that all of the necessary physics has been included. Many physical processes appear to be missing in these simulations, though one does not know for sure how serious any one of them can be. These include non-equilibrium cooling, photo heating, radiative transfer effects, and Pop III star formation, all of which may affect these scaling relations.

Given this uncertain situation, we will follow the analysis in ref. [5], whose data we are adopting in this paper, in which the halo mass was parametrized solely on $z$. This results in constant ratios, as are apparently observed at lower redshifts. These authors argue that a break down of this condition 
at higher redshifts, as suggested by some (perhaps most) simulations, would cause other observational signatures to manifest themselves in the evolution of growth. But no such effects have been observed, lending some support to the assumption of a constant value of these ratios with increasing redshift. These authors considered other possible changes to reconcile the problem, such as varying the Initial Mass Function (IMF), evolving dust corrections, merging and time delays, but to find consistency between theory and observations, they were drawn to unphysical conditions, some of which we described in the Introduction. They concluded that to explain the observed mismatch between the inferred and predicted halo mass function, one has to allow for a rapid evolution in the IMF or a halo to stellar mass ratio that evolves very rapidly. This appears to be unrealistic, given that no such features have been verified observationally.

A quantitative assessment of the degree to which our results (discussed below) would change with an alternative choice of ratios shows that an increase in the correlation $M_{H} / M_{\odot} \approx 120 L_{U V} / L_{\odot}$ by $10 \%$ would still permit a reasonable fit (within one order of magnitude) to the halo mass function at high mass and redshift for $R_{\mathrm{h}}=c t$, but it would not improve the situation for $\Lambda$ CDM. A decrease by $40 \%$ of this ratio would still allow a reasonable fit at high redshift for $\Lambda \mathrm{CDM}$, though not for $R_{\mathrm{h}}=c t$. Our results below will show that the differences in outcome between the two models is so large that variations in quantities such as the $M_{H} / M_{*}$ and $M_{H} / M_{\odot} \approx 120 L_{U V} / L_{\odot}$ ratios are unlikely to be responsible for the full measure of tension between $\Lambda \mathrm{CDM}$ and the measured halo mass function.

It is quite evident in figure 1 that the theoretical predictions of $\Lambda \mathrm{CDM}$ are inconsistent with the observational data. The $\Lambda$ CDM model predicts a sharp evolution in the density of massive halos beyond redshift 4 , but as one can see from this figure, the number density of halos is far greater than predicted at these high redshifts. We shall see shortly that, whereas $\Lambda \mathrm{CDM}$ is left without any viable explanation for the measured growth rate and halo mass function, both of these problems are resolved in the $R_{\mathrm{h}}=c t$ cosmology. In the next section, we will discuss the growth of perturbations in $R_{\mathrm{h}}=c t$ and use this result to derive the halo mass function assuming the ellipsoidal collapse model of Sheth \& Tormen [1] for the overdense regions. 


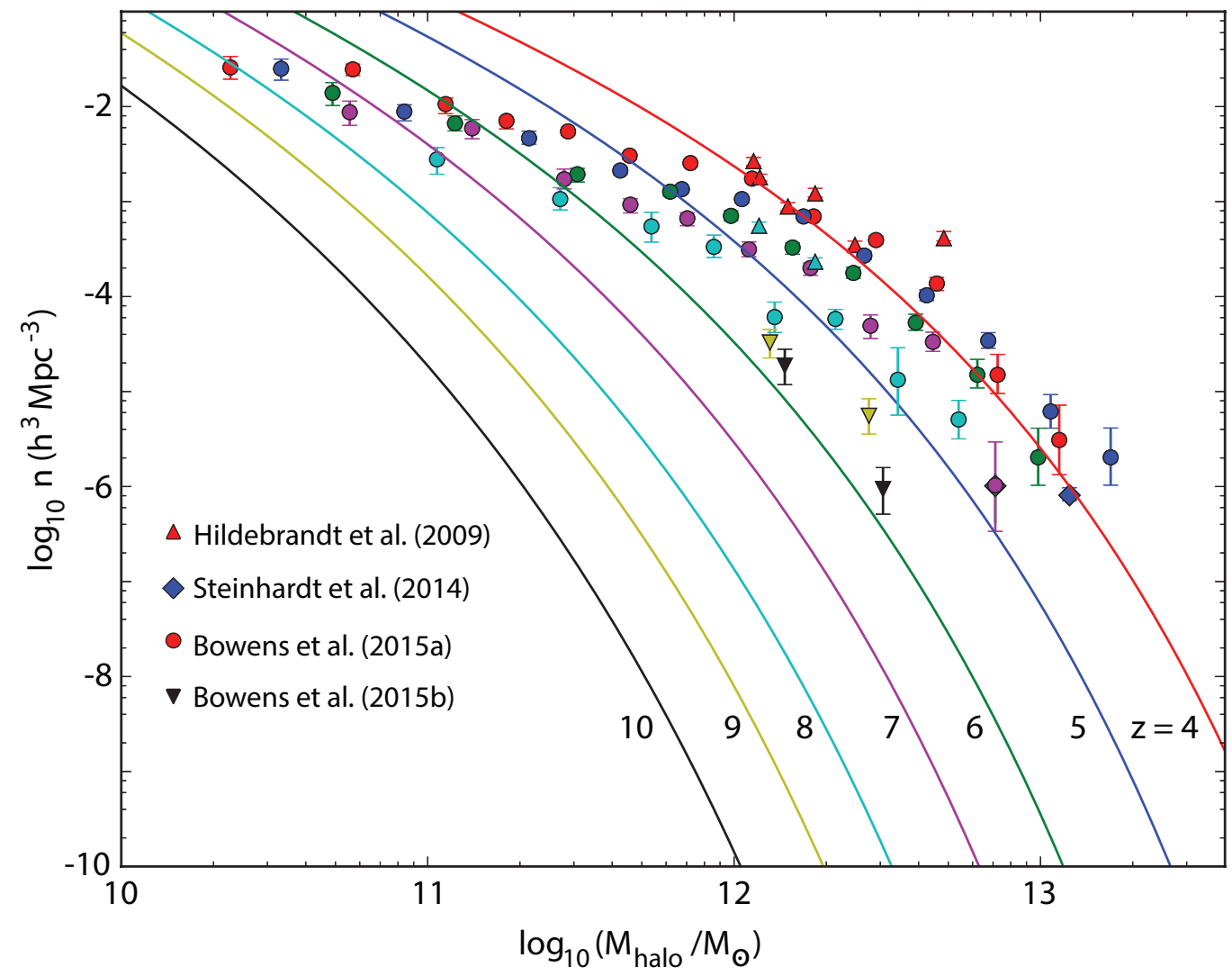

Figure 1: Halo mass function inferred from galaxy surveys, as a function of mass and redshift: $z=4$ (red), 5 (blue), 6 (green), 7 (magenta), 8 (cyan), 9 (yellow), and 10 (black). Solid curves represent the theoretical halo number density predicted by $\Lambda \mathrm{CDM}$ in this same redshift range, based on the estimates of ref. [1] and calculated with the HMFCalc code of ref. [32]. (Adapted from ref. [5]) 


\section{Linear Perturbation Theory and Halo Mass Function in $\boldsymbol{R}_{\mathrm{h}}=c t$}

\subsection{Linear Perturbation Growth}

The initial density field grew as a result of self-gravity, while the Hubble expansion and pressure effects diluted it. Which of these factors dominated at any given time determined the evolution of the initial density fluctuations. To follow the evolution in density, one must solve the relativistic perturbed equations because the matter may be coupled to relativistic components, including radiation. Much of the ground work for this theory has been laid out in ref. [26], and we here adopt some of the principal results of that work to trace out the evolution of the perturbations $\delta \equiv \delta \rho / \rho \leqslant 1$, where the density and pressure are written

$$
\begin{aligned}
& \rho=\rho_{0}+\delta \rho, \\
& p=p_{0}+\delta p .
\end{aligned}
$$

These fluctuations perturb the metric $g_{\alpha \beta}$ and stress-energy tensor $T_{\alpha \beta}$, all of which are included in the set of linearized relations derived from Einstein's equations. Many of the details of this derivation have appeared previously in refs. $[42,43,44,45,46,47,48,49,50,51,52]$. For example, it is not difficult to show that in the covariant Lagrangian approach, where the proper time and cosmic time are related via the gauge transformation

$$
\frac{d \tau}{d t}=1-\frac{\delta p}{\rho+p}
$$

one has $[48,51]$

$$
\frac{d \delta \rho}{d t}=-3 H_{0} \delta \rho-3 \delta H\left(\rho_{0}+P_{0}\right) .
$$

In addition, perturbations to the metric result in

$$
\frac{d \delta H}{d t}+2 H_{0} \delta H+\frac{4 \pi G}{3 c^{2}} \rho_{0} \delta+\frac{v_{s}^{2}}{3(1+w)} D^{2} \delta=0,
$$

where $\delta H$ is the corresponding perturbation to the Hubble constant, i.e., $H(t)=H_{0}(t)+\delta H(t)$, and $w$ is the equation of state parameter, defined as $p=w \rho$. Also, $v_{s}^{2} \equiv \partial p / \partial \rho$ is the sound speed squared.

The $R_{\mathrm{h}}=c t$ cosmology satisfies the zero active mass condition, $\rho+3 p=0$, at all times, so the Universe is dominated by dark energy and (baryonic + 
dark) matter at low redshifts $(z \lesssim 10)$, and by dark energy and radiation in the early Universe. Since we are only considering redshifts $z \lesssim 10$ in this paper, we focus exclusively on the growth of linear perturbations in the matter and dark energy dominated era. We further assume that dark energy acts as a smooth background, so the perturbations are strictly due to fluctuations in the matter density. As discussed in ref. [29], the zero active mass condition requires us to retain the leading second order terms as well, which are usually unimportant relative to the first order terms.

The fluctuation growth is characterized by the following expressions:

$$
\begin{gathered}
\dot{\delta}=-H_{0} \delta-2 \delta H-3 \delta H \delta \\
\ddot{\delta}+3 H_{0} \dot{\delta}-\frac{3}{2}\left(\dot{\delta}^{2}-H_{0} \delta \dot{\delta}+H_{0}^{2} \delta^{2}\right)=v_{s}^{2} D^{2} \delta .
\end{gathered}
$$

As is well known, the second term on the left-hand side represents the dilution due to Hubble expansion, while the third term represents growth due to the gravitational instability. The right-hand side accounts for the effects of pressure within the perturbed fluid, sometimes producing acoustic oscillations. But notice that with the zero active mass condition, the gravitational growth term is zero to first order. This is the most significant difference between the $\Lambda \mathrm{CDM}$ and $R_{\mathrm{h}}=c t$ predictions. While the growth equation in the standard model implies a strong gravitational instability and, therefore, a strong evolution of the halo mass function with redshift (see figure 1), $\delta(t)$, and hence the halo growth rate, are much weaker functions of redshift in $R_{\mathrm{h}}=c t$.

We may find a relatively straightforward solution to Equation (7) by utilizing the weak dependence of $\delta(t)$ on $t$. If we adopt the ansatz

$$
\delta(t) \sim t^{\alpha}
$$

with $|\alpha| \ll 1$, then the third term on the left-hand side of Equation (7) gives

$$
\frac{3}{2}\left(\dot{\delta}^{2}-H_{0} \delta \dot{\delta}+H_{0}^{2} \delta^{2}\right)=\frac{1}{t^{2}}\left(\alpha^{2} \delta^{2}-\alpha \delta^{2}+\delta^{2}\right) \approx B t^{-2} \alpha
$$

(where $B$ is itself much smaller than 1 ), so that

$$
\ddot{\delta}+3 H_{0} \dot{\delta}-\frac{B}{t^{2}} \delta=v_{s}^{2} D^{2} \delta .
$$


We may then follow the conventional procedure of solving this equation using modal analysis with the Fourier decomposition

$$
\delta_{k}(t)=\int \delta\left(x^{\alpha}\right) e^{i \mathbf{k} \cdot \mathbf{x}} d^{3} x
$$

Since, in addition, one has $a(t)=t / t_{0}$ and $H_{0}(t)=1 / t$ in $R_{\mathrm{h}}=c t$, we find that

$$
\frac{d^{2} \delta_{k}}{d t^{2}}+\frac{3}{t} \frac{d \delta_{k}}{d t}-\frac{B}{t^{2}} \delta_{k}=-\frac{k^{2}}{a^{2}} v_{s}^{2} \delta_{k}
$$

In the redshift range $z \lesssim 10$, the Universe is dominated by matter and dark energy, so one may ignore the contribution of radiation and write $\rho \approx \rho_{\mathrm{m}}+\rho_{\text {de }}$. Also, since the perturbation is assumed to contain only matter, $v_{s} \approx 0$, and therefore

$$
\ddot{\delta}_{k}+\frac{3}{t} \dot{\delta}_{k}-\frac{B}{t^{2}} \delta_{k}=0 .
$$

This equation has a polynomial solution,

$$
\delta_{k}(t)=\left(C_{1} t^{-2}+C_{2} t^{B / 2}\right)
$$

where $C_{1}$ and $C_{2}$ are constants that depend on the initial conditions. The first term describes a decaying mode, whereas the second term is the growingactually, quasi-steady-mode. Ignoring the decaying mode, we have

$$
\delta_{k}(t) \approx \delta_{k}\left(t_{0}\right)\left(\frac{t}{t_{0}}\right)^{B / 2}
$$

or, equivalently,

$$
\delta_{k}(z)=\delta_{k}(0)(1+z)^{-B / 2} .
$$

Using a spherical top-hat window function $W_{R}(x)$ to filter the fluctuations $\delta(x)$ on a scale of radius $R$, the variance of the fluctuations is given as

$$
\sigma_{R}^{2}(R, z) \equiv \frac{b^{2}(z)}{2 \pi^{2}} \int_{0}^{\infty} k^{2} P(k) W^{2}(k, R) d k
$$

where $b(z)$ is the usual growth factor which, from Equation (16), may be written as

$$
b(z)=(1+z)^{-B / 2}
$$


The top-hat filter in Fourier-space is given by

$$
W(k, R)=\frac{3[\sin (k R)-k R \cos (k R)]}{(k R)^{3}} .
$$

Since one can readily see from Equation $(9)$ that $|B| \ll 1$, it is clear that the fluctuations grow very slowly at low redshifts. This is the principal feature that makes the predictions of $R_{\mathrm{h}}=c t$ consistent with the $b \sigma_{8}$ data at $z \lesssim 1$, while $\Lambda \mathrm{CDM}$ predicts a significant curvature in this function that is not confirmed by the observations.

\subsection{The Halo Mass Function}

One can address questions concerning the fraction of matter bound in structures and their distribution using the halo mass function, first derived by Press and Schechter [4] assuming spherical collapse and a Gaussian initial density field. It is well known, however, that the Press-Schechter mass function overpredicts the number of high-mass halos and underpredicts the number of low-mass ones. This problem can be resolved using an ellipsoidal collapse model, rather than spherical, producing the Sheth-Tormen mass function [1], given as

$$
f(\sigma)=A \sqrt{\frac{2 a}{\pi}}\left[1+\left(\frac{\sigma^{2}}{a \delta_{c}^{2}}\right)^{p}\right] \frac{\delta_{c}}{\sigma} \exp \left[-\frac{a \delta_{c}^{2}}{2 \sigma^{2}}\right],
$$

where $A=0.3222$ is a normalization constant, $a=0.707$ and $p=0.3$. This mass function is related to the number density of halos with masses less than $M$ as follows:

$$
f(\sigma)=\frac{M}{\rho_{0}(z)} \frac{d n(M, z)}{d \ln \sigma^{-1}},
$$

where $\sigma$ is the variance of the fluctuations in Equation (17).

A quantitative assessment of the merits of Press-Schecter versus ShethTolman is hard to come by because tests of these analytic functions necessarily rely on numerical simulations, rather than actual model-independent measurements. However, in their Bolshoi simulation completed just a few years ago, Klypin et al. [53] examined in detail how the Sheth-Tormen prediction compares with their results as a function of mass and redshift. They found that discrepancies were small, i.e., less than $\sim 10 \%$, at $z \approx 0$ for masses 
in the range $\sim\left(5 \times 10^{9}-5 \times 10^{14}\right) M_{\odot}$. But the Sheth-Tormen analytic function over-predicts the halo abundance at higher redshifts. For example, at $z \sim 6$, Sheth-Tormen over-predicts the number of halos by about $50 \%$ compared to the simulation for masses $\sim 10^{11}-10^{12} M_{\odot}$. This prediction worsens by an order of magnitude at $z \sim 10$.

In the context of $\Lambda \mathrm{CDM}$, a remedy for this defect is to include a multiplicative (correction) factor (introduced in ref. [53]) that brings the ShethTormen approximation to $\lesssim 10 \%$ deviation compared to the simulations for masses $\sim\left(5 \times 10^{9}-5 \times 10^{14}\right) M_{\odot}$, and redshifts $0 \lesssim z \lesssim 10$ :

$$
F(b[z])=\frac{(5.501 b[z])^{4}}{1+(5.500 b[z])^{4}},
$$

where $b(z)$ is the growth factor in Equation (18) (normalized to 1 at $z=0$ ). But as we have pointed out, $b(z)$ is a very slowly-growing function of $z$ in the $R_{\mathrm{h}}=c t$ universe, so the impact of such a correction factor is minimal at best. Since an actual numerical simulation analogous to Bolshoi has not yet been carried out for $R_{\mathrm{h}}=c t$, one does not know yet whether the discrepancies seen for $\Lambda$ CDM persist here. Perhaps the much weaker dependence of $b(z)$ on $z$ mitigates the over-prediction seen for the standard model. For the purpose of this paper, given (1) that the results published in ref. [5] (and reproduced in fig. 1) are based on the Sheth-Tormen approximation without a correction factor, and (2) that the linear growth rate $b(z)$ changes very little with redshift in $R_{\mathrm{h}}=c t$, thus making the correction factor $F(b)$ virtually ineffective, we will continue to use the Sheth-Tormen result in Equation (21) without the possibly important modification introduced in Equation (22). This issue will be revisited in future work once a full numerical simulation analogous to Bolshoi will have been carried out for $R_{\mathrm{h}}=c t$.

In terms of the impact of this approach on the respective results in $\Lambda \mathrm{CMD}$ and $R_{\mathrm{h}}=c t$ are concerned, one can see right away from figure 1 that reducing the Sheth-Tormen prediction for the number of halos at high mass only makes things worse compared to the data shown in this plot, since the standard model's prediction already falls well below the measurements. For $R_{\mathrm{h}}=c t$, on the other hand, we shall see in figure 2 below that the theoretical prediction is very good for the lower masses, but misses progressively more and more towards the high-mass end. The deviation at a halo mass $\sim 10^{13} M_{\odot}$ is a factor of $10-20$, suggestively close to the over-prediction found in [53]. It will be interesting indeed to see in future work if a cor- 
rection factor similar to that in Equation (22) brings the results of $R_{\mathrm{h}}=c t$ completely in line with the data at all masses.

An additionial caveat with our assumptions in this paper is that simulations produce results that are not always consistent with each other when different 'halo finders' are used to identify the mass condensations. The recently performed Bolshoi calculations [53] indicate that Sheth-Tormen (used in our analysis) overpredicts the number of halos found in Bolshoi at $z=8.8$ by a factor of 4-6 when the Spherical Over (SO) density halo finder algorithm is used, whereas Sheth-Tormen is actually consistent with the results based on the Friends of Friends (FOF) algorithm. Worse, the inferred number and mass distributions are inconsistent with each other in the simulation when the results of FOF are compared with those of SO. Bolshoi found that both algorithms identified the same distinct halos, but FOF assigned larger masses to some of them. On average, the masses of halos found with FOF was 1.4 times larger than those based on SO for the same halos. Masses obtained using FOF and SO tend to be consistent at lower redshifts, but deviate from each other at higher redshifts. Noting this as a potential problem to address in future, more elaborate treatments of the analysis we attempt here, we also acknowledge the fact that these inconsistencies are still small compared to other potential systematic issues entering our investigation, such as the poorly known star formation rate and galaxy evolution models used in the 'measurement' of the halo mass function. We will therefore proceed without attempting to address these issues here.

\section{A Cosmological Resolution of the Impossibly Early Galaxy Prob- lem}

Before comparing the predicted halo mass function with the observations, we must recalibrate the data to take into account the differences in differential comoving volume between the two models. This is most easily done using the following expressions for the comoving distance:

$$
D_{\mathrm{com}}^{\Lambda \mathrm{CDM}}=\frac{c}{H_{0}} \int_{0}^{z} \frac{d u}{\sqrt{\Omega_{\mathrm{m}}(1+u)^{3}+\Omega_{\mathrm{r}}(1+u)^{4}+\Omega_{\Lambda}}},
$$

and

$$
D_{\mathrm{com}}^{R_{\mathrm{h}}=c t}=\frac{c}{H_{0}} \ln (1+z) .
$$


Note that here $H_{0}$ refers to the Hubble constant today. The conversion factor to recalibrate the data is then simply

$$
\frac{d V_{\mathrm{com}}^{\Lambda \mathrm{CDM}} / d z}{d V_{\mathrm{com}}^{R_{\mathrm{h}}=c t} / d z}=\left(\frac{D_{\mathrm{com}}^{\Lambda \mathrm{CDM}}}{D_{\mathrm{com}}^{R_{\mathrm{h}}=c t}}\right)^{2} \frac{d D_{\mathrm{com}}^{\Lambda \mathrm{CDM}} / d z}{d D_{\mathrm{com}}^{R_{\mathrm{h}}=c t} / d z} .
$$

The impact of this recalibration may be gauged via a comparison of the data plotted in figures 1 and 2 . Within the redshift range $0 \lesssim z \lesssim 10$, the differential comoving volumes in $R_{\mathrm{h}}=c t$ and $\Lambda$ CDM differ by less than $\sim 10 \%$. One sees similarly small shifts in $n$ between these two plots.

A second factor one must take into account while calculating the halo distribution is the value of $\sigma_{8}(0)$, which is used to normalize the power spectrum. Based solely on fits to the linear growth rate at $z \lesssim 1[26], b \sigma_{8}(0)$ in $R_{\mathrm{h}}=c t$ is inferred to have the value $0.40 \pm 0.03$. The solid curves shown in figure 2 are calculated with the normalizations $b \sigma_{8}(0)=0.37$ and 0.43 , bracketing the $1 \sigma$ variation of the best fit. The consistency between the halo mass function at $z \sim 4-7$ and the linear growth rate at $z \lesssim 1$ [26] should not be underestimated. The fact that these two quite different sets of measurements - the growth rate at $z \lesssim 1$ and the halo mass function at $z \sim 4-7$-are mutually consistent with the expansion and growth rates predicted by $R_{\mathrm{h}}=c t$ is a big factor in favor of this model.

Having said this, it is nonetheless also true that $R_{\mathrm{h}}=c t$ overpredicts the number of halos towards the high mass end, as one can see from the righthand side of figure 2. Several factors may be contributing to this: (1) As noted earlier, the Bolshoi simulation indicates that the Sheth-Tormen mass function overpredicts the number of halos at high mass and high redshift, at least in the context of $\Lambda$ CDM. If an analogous result holds in $R_{\mathrm{h}}=c t$, this might be the cleanest explanation for the theoretical overprediction seen in this figure. To be certain of this effect, however, we must await the completion of a full Bolshoi-like simulation with $R_{\mathrm{h}}=c t$ as the background cosmology. (2) The number of halos inferred at high mass and high redshift may be incomplete due to observational selection effects and/or an incompleteness in the galaxy surveys. In this scenario, the apparent overprediction of halos at high mass and high redshift may get resolved with future observations. (3) As discussed above, the equations we have used in this paper to explore the linear growth rate of fluctuations may break down at $z \gtrsim 10$ due to the increasing role of radiation, which we have ignored until now. A more sophisticated analysis will be required at high redshifts to handle a more 


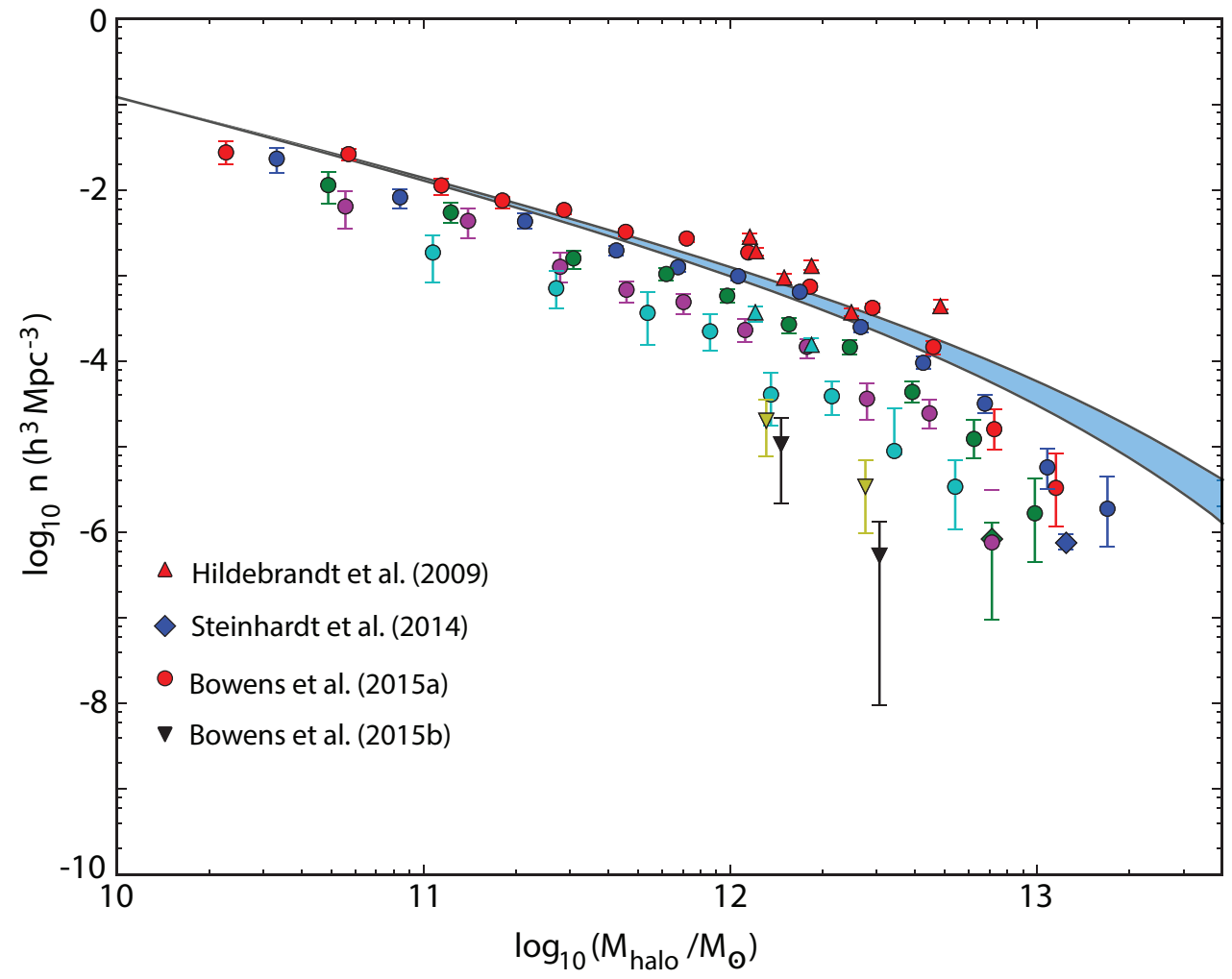

Figure 2: Same as figure 1, except that the data have been recalibrated for the $R_{\mathrm{h}}=c t$ universe using the ratio of differential comoving volumes in Equation (24). The solid curves represent the halo mass function for $R_{\mathrm{h}}=c t$ calculated from Equations (20) and (21), using a normalization $b \sigma_{8}(0)=0.43$ and 0.37 . These two values bracket the $1 \sigma$ variation of the optimized fit to recently published redshift space distortion measurements of the cosmological growth rate [26], which produced the value $b \sigma_{8}(0)=0.40 \pm 0.03$, with $b$ the growth factor in Eq. (18). Given the very weak dependence of $\delta_{k}(z)$ on redshift (Eq. 16), the halo mass function in this model is essentially independent of $z$ in the range $4-10$. 
realistic growth rate calculation.

By comparison, the predicted halo mass function in $\Lambda \mathrm{CDM}$ misses the data everywhere on two counts: first, the shape of $n\left(M_{\text {halo }}\right)$ is not a good match to the observed distribution; $\Lambda$ CDM predicts a steep function, in significant tension with the measurements at all redshifts. Second, $\Lambda C D M$ predicts a strong evolution of the halo mass function with redshift, notably underpredicting the observed density at high redshifts by many orders of magnitude, while at the same time overpredicting their density at lower redshifts. Coupled to the standard model's inability to properly account for the observed flat linear growth rate at $z \lesssim 1$, our results in this paper suggest that the growth of structure in the standard model is inconsistent with the data, at least out to $z \sim 10$.

There are several caveats to this conclusion, however. First, the steepness of the function predicted by $\Lambda \mathrm{CDM}$ is largely due to the assumption of a constant light to mass ratio in the conversion of luminosity to mass. The same assumption does not adversely affect $R_{\mathrm{h}}=c t$, but perhaps the tension with $\Lambda \mathrm{CDM}$ may be alleviated (at least partially) with a more complicated relation between light and mass that produces a shallower mass function. As we have discussed previously, $\Lambda$ CDM does not directly predict this function, relying instead on theoretical adjustments to fit the data. It is more a question of whether these adjustments produce other signatures that may or may not be in tension with the observations. In other words, what see see in figure 1 is essentially a rescaling of the galaxy luminosity function, not a direct measurement of the halo mass function, and this rescaling relies on several pieces of poorly understood physics. Eventually, it will be necessary to carry out a comprehensive simulation that couples the cosmological halo evolution with the physics of galaxy formation in order for us to be more certain regarding the translation of galaxy data into a reliable halo mass function. A second caveat is that even in $R_{\mathrm{h}}=c t$ the halo mass function deviates significantly from the predicted value for $z>7$, as we have discussed above. The fact that both models show significant deviations at the high mass, high redshift end of the halo distribution may therefore be an indication that at least some of the tension with the data is related to the poorly known underlying physics, rather than it merely being an indication that the issue lies solely with the cosmology. 


\section{Conclusion}

Some evidence suggests a disparity between the halo mass function predicted by $\Lambda \mathrm{CDM}$ and the actual measurements of this quantity. This work has been motivated by its lack of anticipated strong evolution in redshift and a significant steepening of the distribution with increasing mass. The observed halo mass function is relatively flat, pointing to a much higher density at the high-mass end, and a correspondingly lower density of halos with smaller masses. In addition, the halo distribution appears to be evolving much more slowly than is required by the standard model, at least for redshifts $z \lesssim 10$.

In earlier work, we used recently published redshift space distortion measurements of the cosmological growth rate, $b \sigma_{8}(z)$, at redshifts $z \lesssim 1$, to show that the linear evolution of perturbations in the $R_{\mathrm{h}}=c t$ cosmology appears to be a better match to the data than the current standard model. In that work, we found an optimized growth rate $b \sigma_{8}(0)=0.40 \pm 0.03$. Interestingly, the halo mass function in $R_{\mathrm{h}}=c t$, normalized using this value, fits the measured halo distribution very well, certainly much better than $\Lambda$ CDM does. Of course, one must still be wary of these results, given that the halo masses cannot be measured directly. Nonetheless, since the various techniques used to translate the observed galaxy mass distribution into a halo mass function all agree qualitatively on the outcome, the mutual consistency between the halo fits at $z \sim 4-10$ and the linear growth rate fit at $z \lesssim 1$, suggests that the formation of structure predicted by $R_{\mathrm{h}}=c t$ is a better fit to the observations than that expected in $\Lambda \mathrm{CDM}$.

Although our focus in this paper has been exclusively on the formation and evolution of halos, the surprising nature of the halo mass function has also been characterized as a breakdown in the theory of galaxy formation at high redshifts. But this conclusion is actually not new. It has been known for several years that the observed high- $z$ quasars and galaxies must have formed much too quickly when viewed with the timeline afforded them by the standard model $[21,23,22]$. For example, in order to understand the emergence of $\sim 10^{9} M_{\odot}$ black holes earlier than $z \sim 6-7$ in $\Lambda$ CDM, one must assume that black-hole growth either began with anomalously large seeds $\left(M>10^{5} M_{\odot}\right)$, or proceeded at super-Eddington rates, neither of which has ever been seen-here locally, or at high redshifts. Yet in $R_{\mathrm{h}}=c t$, the timeline is just right to have allowed these high- $z$ objects to form according to well known and understood astrophysical principles. 
Our analysis of the halo mass function in this paper constitutes another important confirmation of the expansion scenario predicted by the $R_{\mathrm{h}}=c t$ cosmology. But there is much work yet to be done with the formation of structure in this model. Though $R_{\mathrm{h}}=c t$ accounts for the observed halo distribution very well for masses $M \lesssim 10^{12} M_{\odot}$, its predictions deviate from the observations by one to two orders of magnitude at the highest mass end. Though this is still much less extreme than the situation one confronts in $\Lambda \mathrm{CDM}$, this divergence suggests several possible explanations that need to be explored. These include (1) the linear growth rate seen at low redshifts in $R_{\mathrm{h}}=c t$ may need modification at $z \gtrsim 10$. This would not be surprising, given that the current theory is based on the dominance of matter and dark energy in the cosmic fluid. But at high redshifts, the growing relevance of radiation cannot be ignored; and (2) The relations used to infer the halo masses from large galaxy surveys ought to be revisited. Attempts at mitigating the disparity between theory and observation in $\Lambda$ CDM had very little impact. They may be more successful in the case of $R_{\mathrm{h}}=c t$, since the current tension exists solely for $M \gtrsim 10^{12} M_{\odot}$. The halo mass function for masses lower than this agrees with theory very well.

Eventually, a complete study of galaxy formation and clustering in $R_{\mathrm{h}}=$ ct needs to be carried out in concordance with the evolution of the halo mass function. Hopefully, this self-consistent approach will mitigate at least several of the uncertainties still hindering the interpretation of halo properties from the actual measurement of the galaxy luminosity function and spectrum. Currently, there is still too much freedom in combining various physical influences on galaxy evolution to arrive at a unique picture of structure formation at redshifts $z \lesssim 10$.

\section{Acknowledgments}

We are very grateful to the anonymous referee for suggesting several important improvements to the presentation of our results in this paper. FM is grateful to the Instituto de Astrofísica de Canarias in Tenerife and to Purple Mountain Observatory in Nanjing, China for their hospitality while part of this research was carried out. FM is also grateful for partial support to the Chinese Academy of Sciences Visiting Professorships for Senior International Scientists under grant 2012T1J0011, and to the Chinese State Administration of Foreign Experts Affairs under grant GDJ20120491013. 


\section{References}

[1] R. K. Sheth, H. J. Mo and G. Tormen, MNRAS 3231 (2001) 1.

[2] V. Springel, S.D.M. White, A. Jenkins et al., Nature 435 (2005) 629.

[3] M. Vogelsberger, S. Genel, V. Springel et al., MNRAS 444 (2014) 1518.

[4] W. Press and P. Schechter, ApJ 187 (1974) 425.

[5] C. L. Steinhardt, P. Capak, D. Masters and J. S. Speagle, ApJ 824 (2016) 1.

[6] N. A. Grogin, D. D. Kocevski, S. M. Faber et al., ApJS 197 (2011) 35.

[7] A. M. Koekemoer, S. Faber, H. Ferguson et al., ApJS 197 (2011) 36.

[8] P. Capak et al., in preparation (2016).

[9] H. Hildebrandt, J. Pielorz, T. Erben et al., A\&A 498 (2009) 725.

[10] K.-S. Lee, H. C. Ferguson, T. Wiklind et al., ApJ 752 (2012) 66.

[11] O. Ilbert, H. J. McCracken, O. Le Févre et al., A\&A 556 (2013) A55.

[12] S. L. Finkelstein, M. Song, P. Behroozi et al., ApJ 814 (2015) 95.

[13] K. I. Caputi, O. Ilbert, C. Laigle et al., ApJ 810 (2015) 73.

[14] C. G. Lacey, C. M. Baugh, C. S. Frenk, and A. J. Benson, MNRAS 412 (2011) 1828.

[15] K. Finlator, B. D. Oppenheimer, and R. Davé, MNRAS 410 (2011) 1703.

[16] D. Waters, S. M. Wilkins, T. Di Matteo, Y. Feng, R. Croft and D. Nagai, MNRAS 461 (2016) L51.

[17] P. S. Behroozi and J. Silk, ApJ 799 (2015) id 32.

[18] N. Mashian, P. A. Oesch and A. Loeb, MNRAS 455 (2016) 2101.

[19] R. J. Bouwens, G. D. Illingworth, P. A. Oesch et al., ApJ 803 (2015) 34. 
[20] R. J. Bouwens, P. A. Oesch, I. Labbe et al., ApJ 830 (2015) id 67.

[21] F. Melia, ApJ 764 (2013) 72.

[22] F. Melia and T. M. McClintock, Proc. R. Soc. A 471 (2015) 20150449.

[23] F. Melia, AJ 147 (2014) 120.

[24] A. Leauthaud, J. Tinker, K. Bundy et al., ApJ 744 (2012) 159.

[25] Planck Collaboration, P.A.R. Ade, N. Aghanim et al., A\&A 594 (2016) id A13.

[26] F. Melia, MNRAS 464 (2017) 1966.

[27] F. Melia, MNRAS 382 (2007) 1917.

[28] F. Melia, Frontiers of Phys. 11 (2016) 118901.

[29] F. Melia, Frontiers of Phys. 12 (2017) 129802.

[30] F. Melia and M. Abdelqader, IJMP-D 18 (2009) 1889.

[31] F. Melia and A. Shevchuk, MNRAS 419 (2012) 2579.

[32] S. G. Murray, C. Power and A.S.G. Robotham, A\&C 3 (2013) 23.

[33] C. Conroy, J. E. Gunn, and M. White, ApJ 699 (2009) 486.

[34] D. Schaerer and S. de Barros, A\&A 515 (2010) A73.

[35] D. P. Stark, M. A. Schenker, R. Ellis, B. Robertson, R. McLure and J. Dunlop, ApJ 763 (2013) 129.

[36] J. S. Speagle, C. L. Steinhardt, P. L. Capak and J. D. Silverman, ApJ 214 (2014) 15.

[37] Ma Xiangcheng, Philip F. Hopkins, Shea Garrison-Kimmel et al., arxiv:170606605M (2017)

[38] Mauro Stefanon, Rychard J. Bouwens, Ivo Labbé, ApJ 843 (2017) 36S.

[39] Daniel Ceverino, Simon C.O.Glover, Ralf S. Klessen, MNRAS 470 (2017) 2791C. 
[40] Brian W.Oshea, John H. Wise, Hao Xu, et al., ApJ 807 (2015) 12 O.

[41] Trac H., Cen R., Mansfield P., 2015, ApJ, 813, 54T.

[42] S. Weinberg, Gravitation and Cosmology, Wiley: NY (1972).

[43] L. D. Landau and E. M. Lifshitz, The Classical Theory of Fields, Pergamon Press: Oxford (1975).

[44] P.J.E. Peebles, The Large-scale Structure of the Universe, Princeton University Press: NY (1980).

[45] P.J.E. Peebles, Principles of Physical Cosmology, Princeton University Press: NY (1993).

[46] W. H. Press and E. T. Vishniac, ApJ 239 (1980) 1.

[47] E. W. Kolb and M. Turner, The Early universe, Addison-Wesley: Reading MA (1990).

[48] T. Padmanabhan, Structure Formation in the Universe, Cambridge University Press: Cambridge, England (1993).

[49] P. Coles and F. Lucchin, Cosmology: The Origin and Evolution of Cosmic Structure, Wiley: NY (1995).

[50] J. A. Peacock, Cosmological Physics, Cambridge University Press: Cambridge, England (1999).

[51] A.R.D. Liddle and K. A. Lyth, Cosmological Inflation and Large-Scale Structure, Cambridge University Press: Cambridge, England (2000).

[52] C. G. Tsagas, A. Challinor and R. Maartens, Phys. Reports 465 (2008) 61.

[53] Klypin, A. A., Trujillo-Gomez, S. \& Primack, J., ApJ 740 (2011) 102. 